\title{
DFT study of photoelectric properties of some organic polymers
}

Abstract

Polyacetylens noted PA, with general formulae $(-\mathrm{CH}=\mathrm{CH}-)_{n}$ are organic compounds with double conjugated bonds. They are the thirst polymers conducting characters which, after doping, were highlighted. Indeed, the semiconducting character of an organic material resides in the alternation of single and double covalent bonds; the $\pi$ bonds electrons, in these conditions, are able to be delocalization. That delocalisation assures the transport of charges. The substitution derivatives of these compounds may exhibit better conduction capabilities. Since AP and their derivatives, like many other organic conjugated materials, absorb electromagnetic waves in the visible range of the spectrum, they are used as photovoltaic materials, essential parts of organic solar cells. The present work focused on the theoretical study of the evolution of the photovoltaic character of PA and their halogenated derivatives as a function of their polymerization index ( $\mathrm{n}, \mathrm{n}=1$ to 5 ); this study was carried out consideration the energy gaps between the LUMO and the HOMO of the molecules involved in this work and confirmed the experimental results known in the literature.

The calculations were performed by the DFT/B3LYP method in the $6-31 \mathrm{~g}+(\mathrm{d}, \mathrm{p})$ orbital basis. The Gaussian program in its versions 98,03 and 09 was used to perform these calculations on the HPL1908w, Samsung Intel ${ }^{\circledR}$ Core i3-3110 CPU and Dell computers. After comparing the differences between the LUMO of the molecules, it has been proposed to use photovoltaic "donor-acceptor" combinations based on anthracene and polyacetylene.

Keywords: polyacetylens, Anthracene, photovoltaic conjugated organic materials, LUMO, HOMO, DFT/B3LYP, Base 6-31g+(d,p)
Volume I Issue 4 - 2017

Kuevi Urbain Amah

Department of Chemistry,Abomey-Calavi University, Benin

Correspondence: Kuevi Urbain Amah, Laboratory of

Theoretical Chemistry and Molecular Spectroscopy

(LACTHESMO), Department of Chemistry, Abomey-Calavi

University, Benin, 03 BP: 3409 Cotonou Bénin and 0I BP 1326

Cotonou Benin, Tel (+229)950-699-02, 660-302-97,

Email, urviama@gmail.com

Received: September 15, 2017 | Published: October 03, 2017
Abbreviations: LUMO, lowest unoccupied molecular orbital; HUMO, highest occupied molecular orbital; D/A, donor-acceptor; PPP, poly (para-phenylene); PT, poly thiophene; PA, poly acetylene

\section{Introduction}

Given the dizzying growth in global energy needs and the surge in crude oil prices, the diversification of energy sources and, above all, the use of renewable sources of energy that respects the environment are increasingly emerging as the best alternative to curbing the crisis. ${ }^{1}$ Among the renewable energy sources the solar energy, practically inexhaustible, is by far the most available natural source of energy, clean and accessible at every point of the earth's surface. ${ }^{1,2}$ It can therefore be an excellent solution to the problem of energy supply in the world and especially in the sunny geographical areas of the globe.

The $99 \%$ of the photovoltaic cells produced today in the world, to tame and to use the solar energy, are based on silicon, material of which the growing demand in the electronic field raises the problem of its availability. Moreover, the production of this material requires truly polluting and costly purification processes which make it impossible for ordinary people to acquire and use silicon-based solar panels as a source of energy. ${ }^{1}$ This is why, worldwide, research teams are working to find other materials with interesting semiconductor properties. In this stride, organic molecules or polymers are increasingly explored and used as semiconductor materials in the design of photovoltaic cells. However, in this scientific endeavor, one of the challenges still remains the improvement of the electrical conversion efficiency of these organic semiconductors. ${ }^{1-3}$ The semiconducting properties of organic materials come from the alternation of single and double covalent bonds in their molecular structures. ${ }^{1-5}$ This electronic conjugation which favors the delocalisation and the circulation of the electronic charges can then constitute a first criterion for the selection of the molecules likely to be involved in the design of an organic photovoltaic cell. The photovoltaic effect is a phenomenon which consists in generating electricity by absorption and conversion of light energy into electrical energy. An organic solar cell consists of a layer of photoactive organic materials sandwiched between two electrodes: $:^{1,2}$

i. The active layer consists, on the one hand, of electron-donating organic molecules (D), possessing a low ionization potential and permitting the transport of holes, and, on the other hand, of organic electron accepting molecules (A), having a high electron affinity and enabling the electron transport.

ii. The anode, generally composed of indium and tin oxide, is deposited on a glass or transparent plastic substrate,

iii. The cathode, traditionally composed of metals with low extraction activity, is deposited by evaporation on the active layer.

The phenomenon of conversion of light into electricity in organic photovoltaic cells is a complex process combining the absorption of photons in the active layer, the diffusion of the excitons formed, their dissociation as well as the transport and collection of charges -5]. The 
prediction of the optimum conditions for carrying out such a process can theoretically be achieved on the basis of certain molecular, electronic and spectroscopic parameters such as molecular orbital's energies, ionization potential, electronic affinity, intermolecular distances, photonic absorption bands, etc., parameters whose theoretical values can be determined by methods of quantum chemistry. ${ }^{6}$

In fact, photons emitted by the sun, whose maximum luminous power is observed in the range of 450 to $1000 \mathrm{~nm}$, are absorbed by the active layer. This absorption of photons leads to the formation of an excitons by the passage of an electron from the highest occupied molecular orbital (HOMO) of the donor to its lowest unoccupied molecular orbital (LUMO). The electronic transfer of the LUMO from the donor to the LUMO of the acceptor is all the more likely as the energy difference between these two levels is great. This energy difference must be greater than $0.4 \mathrm{eV}$ for dissociation to take place. In addition, the transfer of the LUMO from the donor to the LUMO of the acceptor must be faster than the recombination process. To avoid electronic transfer accompanied by a hole transfer from the donor to the acceptor, the HOMO level of the donor must also be higher than that of the acceptor so that the energy barrier is impassable by the hole. The transfer of charge to the Donor-Acceptor interface $(\mathrm{D} / \mathrm{A})$ is in competition with excitons recombination; only the excitons generated at a distance sufficiently close to the interface can reach the interface and be dissociated there before recombining. The critical distance is of the order of the diffusion length of the excitons (a few nanometers). ${ }^{1-5}$

*Dopage de type n:

$$
(\pi \text { - polymère })_{n}+\left[\mathrm{Na}^{+}\left(\mathrm{C}_{10} \mathrm{H}_{8}\right)^{-\cdot}\right]_{y} \rightarrow\left[\left(\mathrm{Na}^{+}\right)_{y}(\pi \text {-polymère })^{-y}\right]_{n}+\left(\mathrm{C}_{10} \mathrm{H}_{8}\right)^{0} \cdot^{4}
$$

The introduction of charges during the doping process, locally modifies the alternation of the single and double bonds, thus positioning the charge carriers on the chain. In the forbidden band some new electronic states appeared and located with holes (radical cations) or electrons. The quasi-particles thus formed can be classified into two categories: solitons on the one hand, polarons and bi polarons on the other hand.

-When a double bond breaks (rupture of a $\pi$ bond), it can give rise to two radicals called solitons and anti-solitons which can move away from each other by alternation of the links giving two independent solitons on the chain. This process of creating solitons/anti-solitons pairs is thermally activated and facilitated by defects on the polymer chain. Solitons create boundaries between phases A and B of the same energy but different by the configuration of the links alternation (Figure 1)

-A conjugated polymer may interact with an electron acceptor compound (A), which may result in electron transfer of the polymer to the acceptor. The obtained species is a polymer having a positive charge on the chain, with, in the vicinity, a counter-anion (Figure 2).

The alternation between double and single links is locally scrambled. The cationic radical so corresponds to a defect delocalized on several carbon atoms. This deformation stabilizes the charge since it accompanies it in its movement. The moving species made up of the charge-deformation set is called positive polarons. The conjugated polymer may interact with an electron donor compound (D), which may result in an electron transfer from the donor to the polymer. The
The obtaining in 1977 of polyacetylene having a conductivity of the order of $10^{3} \mathrm{~S} / \mathrm{cm}$, by doping (with $\mathrm{Br}_{2}, \mathrm{I}_{2}$ or $\mathrm{AsF}_{5}$ ), triggered a real craze for this material. However, this has a low environmental and thermal stability limiting its technical use. As a result of these observations, many conjugated aromatic polymers have been studied such as poly (para-phenylene) (PPP), Polythiophene (PT)...,. Polypyrrole.

In order to improve the mechanical and conductive properties of the polymers, filler can be introduced into the structure of the semiconductor polymer by a so-called doping process. This process consists of an oxidation-reduction reaction by introducing impurities (doping type $n$ ) or acceptor (p-type doping) in the material and is mainly carried out chemically or electrochemically. It is, however, important to note that unlike conductive polymers, semiconductors do not always have reversible and easily controllable doping. In the case of chemical doping, for example, for p-type (or n-type) doping, the neutral conjugated polymer is converted into a poly (cation) (or poly (anion)) by the action of an oxidant or reducing). At the same time, a counter ion is associated with it in order to maintain the overall electro-neutrality of the system, as illustrated in the following examples:

$$
\begin{aligned}
& \text { *P-type doping: } \\
& (\pi \text {-polymère })_{n}+3 / 2 n y\left(I_{2}\right) \rightarrow\left[(\pi \text { - polymère })^{+y}\left(I_{3}^{-}\right)_{y}\right]_{n}
\end{aligned}
$$

obtained species is a polymer having a negative charge on the chain, with, in the vicinity, a counter cation. The negatively charged moving species is called the negative polarons. If we remove (or add) a second electron to the existing polarons, a new species can be created: it is a bipolarons; bipolarons is a di cation or dianion. The bipolarons has a null spin.

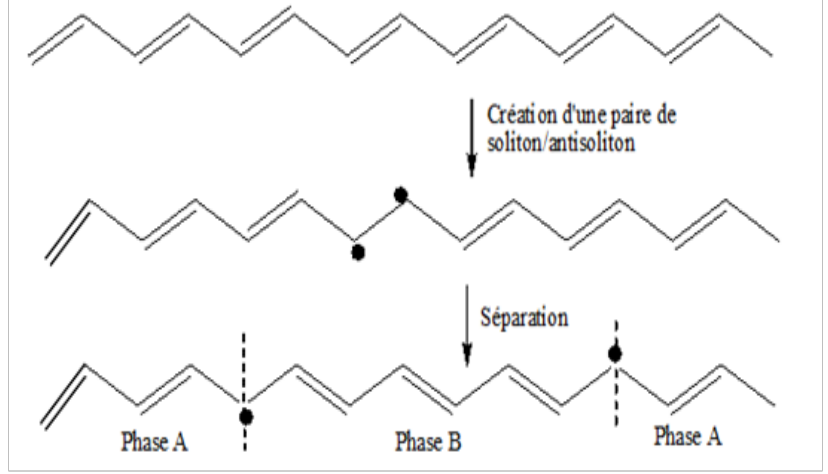

Figure I Solitons formation mechanism.

The installed capacity of the global photovoltaic fleet increased six-fold between the end of $2010(50 \mathrm{GW})$ and the end of $2016(305 \mathrm{GW}) .^{6}$ In recent years there has been a renewed interest in the field of organic photovoltaic converters. Research efforts have been multiplied, supported by budgets from a few industries and development of renewable energies. Recently, yields of over $4 \%$ have been reported in publications and prototypes with about $5 \%$ were 
manufactured for $100 \%$ organic solar cells. These progress suggests that future commercialization of these devices can be envisaged. ${ }^{7}$ The present study has examined the theoretical study of the photovoltaic characters of polyacetylene PA, of general formula

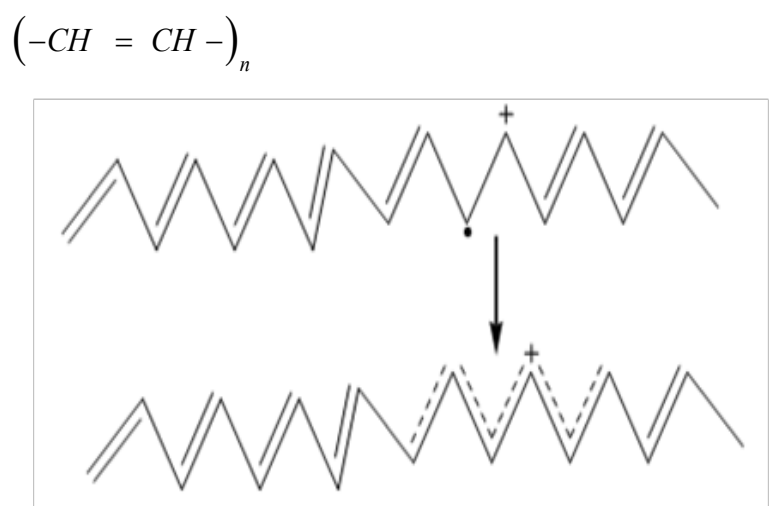

Figure 2 Formation of a positive polarons.

$(n=1$ to 5$)$ and their halogenated and alkaline derivatives. This work has also taken into account aromatic compounds. PA is the first polymers whose conducting character, after doping, was highlighted. The substitution derivatives of these compounds may exhibit better electrical conduction capability. This study was carried out consideration the energy gaps between the LUMO and the HOMO of the molecules involved in this work and confirmed the experimental results known in the literature. After comparing the differences between the LUMO of the molecules, it has been proposed to use photovoltaic "donor-acceptor" combinations based on anthracene and polyacetylene. The present studies were carried out theoretically by adopting the DFT/B3LYP density functional method, one of the most widely used methods in quantum computations of the matter structure.

\section{Materials and methods}

In the present work the calculations were carried out by the DFT method with the functional B3LYP. The main aim of the DFT is to replace the multi-electron wave function by the electronic density. While the multi electronic wave function depends on $3 \mathrm{~N}$ variables (where $\mathrm{N}$ is the total number of particles in the system), density is only a function of three variables; it is therefore an easier quantity to treat both mathematically and conceptually. The principle of DFT consists of a reformulation of a N-body problem into a single-body problem, or, two-body problem if one considers the spin. The central idea of DFT is that the electron density of the fundamental state of the system determines the average values of observables, such as energy. ${ }^{8,9}$ The functional B3LYP is the most used by chemists. This signifies that the exchange energy is calculated by the Becke method with three parameters and the correlation by the method of ${ }^{1-18}$ Lee et al. ${ }^{11}$

The calculations were performed by the DFT/B3LYP method in the $6-31 \mathrm{~g}+(\mathrm{d}, \mathrm{p})$ orbital basis. The Gaussian program in its versions 98, 03 and $09^{12}$ was used to perform these calculations on the HPL1908w, Samsung Intel $®$ Core i3-3110 CPU and Dell computers. The molecular representations were carried out using the Gauss View 3.09 software, the graphs by the Excel 2007 software.

\section{Results and discussion}

The first part of our calculations was devoted to the study of the conductivity of PA and their derivatives. Table 1 summarizes the energy parameters related to the photoconductivity of the molecules.

Table I Energy parameters related to the photoconductivity of some conjugated organic molecules

\begin{tabular}{|c|c|c|c|c|c|c|c|c|c|}
\hline \multicolumn{5}{|l|}{ Polyacetylenes } & \multicolumn{5}{|c|}{ Halogenated derivatives } \\
\hline Molecule & $\begin{array}{l}\text { LUMO } \\
\text { (u.a.) }\end{array}$ & $\begin{array}{l}\text { HOMO } \\
\text { (u.a.) }\end{array}$ & $\begin{array}{l}\text { Gap } \\
\text { (u.a.) }\end{array}$ & $\begin{array}{l}\text { Gap } \\
(\mathrm{eV})\end{array}$ & $\begin{array}{l}\text { Nom de la } \\
\text { molécule }\end{array}$ & $\begin{array}{l}\text { LUMO } \\
\text { (u.a.) }\end{array}$ & $\begin{array}{l}\text { HOMO } \\
\text { (u.a.) }\end{array}$ & $\begin{array}{l}\text { Gap } \\
\text { (u.a.) }\end{array}$ & $\begin{array}{l}\text { Gap } \\
\text { (eV) }\end{array}$ \\
\hline \multirow{2}{*}{ Ethylene } & -0.00 & -0.278 & 0,268 & 7,313 & \multirow{2}{*}{ Fluoro Ethylene } & -0.008 & -0.277 & 0,269 & 7,322 \\
\hline & 952 & 27 & 75 & & & 34 & 45 & 11 & \\
\hline \multirow{2}{*}{ Butadiene } & -0.041 & -0.24 & 0,198 & 5,412 & \multirow{2}{*}{ Chloro Ethylene } & -0.022 & -0.271 & 0,248 & 6,754 \\
\hline & 48 & 37 & 89 & & & 91 & 12 & 21 & \\
\hline \multirow{2}{*}{ Hexatriene } & -0.06 & -0.22 & 0,160 & 4,366 & \multirow{2}{*}{ Bromo Ethylene } & -0.025 & -0.264 & 0,239 & 6,504 \\
\hline & 05 & 51 & 46 & & & 48 & 51 & 03 & \\
\hline \multirow{2}{*}{ Octatetrene } & -0.067 & -0.21 & 0,143 & 3,91 & & & & & \\
\hline & 00 & 67 & 67 & & & & & & \\
\hline \multirow{2}{*}{ Decapentene } & -0.08 & -0.199 & 0,119 & 3,26 & \multirow{2}{*}{ Fluoro Butadiene } & -0.043 & -0.241 & 0,197 & 5,382 \\
\hline & 01 & 79 & 78 & & & 69 & 48 & 79 & \\
\hline \multirow{4}{*}{$\begin{array}{l}\text { Pentabromo } \\
\text { acetylene }\end{array}$} & -0.11 & -0.22 & 0,11 & 2,994 & \multirow{2}{*}{ Chloro Butadiene } & -0.05 & -0.24 & 0,190 & 5,177 \\
\hline & 086 & 090 & 004 & $2 I(A)$ & & 65 & 91 & 26 & \\
\hline & & & & & \multirow{2}{*}{ Bromo Butadiene } & -0.054 & -0.240 & 0,186 & 5,073 \\
\hline & & & & & & 27 & 70 & 43 & \\
\hline \multirow{2}{*}{ Lithium Ethylene } & -0.049 & -0.191 & 0,142 & 3,874 & \multirow{2}{*}{ Lithium Butadiene } & -0.052 & -0.194 & 0,142 & 3,870 \\
\hline & 45 & 83 & 38 & 3 & & 4 & 28 & 24 & \\
\hline
\end{tabular}


Table Continued...

\begin{tabular}{|c|c|c|c|c|c|c|c|c|c|}
\hline \multicolumn{5}{|l|}{ Polyacetylenes } & \multicolumn{5}{|c|}{ Halogenated derivatives } \\
\hline Molecule & $\begin{array}{l}\text { LUMO } \\
\text { (u.a.) }\end{array}$ & $\begin{array}{l}\text { HOMO } \\
\text { (u.a.) }\end{array}$ & $\begin{array}{l}\text { Gap } \\
\text { (u.a.) }\end{array}$ & $\begin{array}{l}\text { Gap } \\
(\mathrm{eV})\end{array}$ & $\begin{array}{l}\text { Nom de la } \\
\text { molécule }\end{array}$ & $\begin{array}{l}\text { LUMO } \\
\text { (u.a.) }\end{array}$ & $\begin{array}{l}\text { HOMO } \\
\text { (u.a.) }\end{array}$ & $\begin{array}{l}\text { Gap } \\
\text { (u.a.) }\end{array}$ & $\begin{array}{l}\text { Gap } \\
\text { (eV) }\end{array}$ \\
\hline \multirow{2}{*}{ Sodium Ethylene } & -0.062 & -0.177 & 0,115 & 3,134 & \multirow{2}{*}{ Sodium Butadiene } & -0.064 & -0.189 & 0,125 & 3,405 \\
\hline & 34 & 53 & 19 & & & 38 & 52 & 14 & \\
\hline \multirow{2}{*}{ Potassium Ethylene } & -0.056 & -0.149 & 0,092 & 2,528 & \multirow{2}{*}{$\begin{array}{l}\text { Potassium } \\
\text { Butadiene }\end{array}$} & -0.058 & -0.162 & 0,104 & 2,832 \\
\hline & 40 & 33 & 93 & 7 & & 44 & 52 & 8 & \\
\hline \multirow{2}{*}{ Benzene } & -0.015 & -0.257 & 0,242 & 6,589 & \multirow{2}{*}{$\begin{array}{l}\text { Hexatriene Dop } \\
\text { Br- }\end{array}$} & 0.113 & 0.008 & 0,105 & 2,86 \\
\hline & 47 & 62 & 15 & & & 31 & 21 & I & \\
\hline \multirow{2}{*}{ Biphenyle } & -0.039 & -0.233 & 0,193 & 5,272 & \multirow{2}{*}{$\begin{array}{l}\text { Tribromo } \\
\text { Acetylene }\end{array}$} & -0.08 & -0.235 & 0,14 & 4,028 \\
\hline & 73 & 46 & 73 & & & 739 & 42 & 803 & \\
\hline \multirow{2}{*}{ Naphtalene } & $-0,049$ & $-0,223$ & 0,174 & 4,735 & \multirow{2}{*}{$\begin{array}{l}\text { Tribromo } \\
\text { Acetylene Dop Br }\end{array}$} & -0.128 & -0.21 & 0,08 & 2,38 \\
\hline & 63 & 64 & I & & & 78 & 641 & 763 & \\
\hline \multirow{2}{*}{ Anthracene } & $-0,072$ & $-0,202$ & 0,130 & 3,54 & \multirow{2}{*}{$\begin{array}{l}\text { Tribromo } \\
\text { Acetylene Dop K }\end{array}$} & -0.11 & -0.12 & 0,013 & 0,37 \\
\hline & 70 & 78 & 08 & (D) & & 76 & 434 & 58 & \\
\hline
\end{tabular}

The analysis of the HOMO to LUMO gaps in the polyacetylene series shows that the value of this quantity decreases as the carbon chain lengthens. It varies from 7,313 to 3,259eV. This confirms the fact that delocalization increases with the number of conjugations, which increases the conductivity of the material $;{ }^{1-5}$ in fact the passage from the HOMO to the LUMO is facilitated when the energy barrier to be crossed is weak. The calculated values of the energy gaps correspond to the ultraviolet and visible domains as has been discovered experimentally. This UV-visible absorption property justifies the use of polyacetylene (PA) as photovoltaic materials.

In the search for substituent's capable of improving the conductivity of polyacetylene, the present work has been interested in studying the influence of halogens on the one hand and alkalies on the other (two families of the most active chemical elements) on the conductivity of organic materials. Examining the results in Table 1, it can be seen that the improvement in conduction of the PA material increases markedly from fluorine to iodine as a substituent. This may be explained by the fact that the peripheral free electron orbital's $p$ of the halogens are increasingly energies from F to I; the capacity of these orbital's to enter into conjugation with the neighboring $\pi$ bonds is then reinforced from light halogens to heavy halogens. The same tendency is observed from the alkali metal of the first period of the classification table (lithium) to that of the third period (potassium). The finding in the case of alkalies is explained by the fact that the number of empty orbitals (i.e. the number of holes) increases from $\mathrm{Li}$ to $\mathrm{K}$, and certainly beyond; the holes facilitate the delocalization of the $\pi$ electrons of the PAs and, moreover, that of the conjugated bonds of the other organic polymers with conjugated double bonds.

Table 1, showing the energetic values of LUMO and HOMO of benzene, biphenyl, naphthalene and anthracene (gaps=6.589, 5.272, 4.735 and $3.54 \mathrm{eV}$ respectively) also showed that the conjugation increases when, increases the number of aromatic rings, which means that the conjugation increases with the number of conjugated $\pi$ bonds.

Following these observations, it appeared that the brominated polymers, in this case the poly (monobromo) acetylenes, must exhibit interesting electronic conduction properties. We have therefore studied pentabromoacetylene (PBA) of formula

$\mathrm{CH}_{2}=\mathrm{CBr}-\mathrm{CH}=\mathrm{CBr}-\mathrm{CH}=\mathrm{CBrH}-\mathrm{CH}=\mathrm{CBr}-\mathrm{CH}=\mathrm{CHBr}$

brominated derivative of decapentaene

$$
\mathrm{CH}_{2}=\mathrm{CH}-\mathrm{CH}=-\mathrm{CH}=\mathrm{CH}_{2}
$$

It was found that the introduction of the five bromine atoms had a bathochromic effect since the energy gap increased from 3.26 (for decapentaene) to $2.994 \mathrm{eV}$ (for PBA). The gap being reduced the conduction of the material would be improved. In order to propose a combination of materials which can serve as an active layer in a photovoltaic cell, the values of different energies taking into account in the electrical conductivity of the materials were compared. From this comparison it appeared that the combination Anthracene/PBA would be indicated as a photovoltaic active layer (Figure 3) (Figure 4).

Indeed the difference $\mathrm{LUMO}_{\text {Anthracene }}-\mathrm{LUMO}_{\text {Pentabromoacetylene }}$ was found to equal $1.038 \mathrm{eV}$; this value is much greater than the threshold value $(0.4 \mathrm{eV})^{1}$ favorable to the dissociation of the excitons. In the Anthracene/PBA mixture, anthracene will serve as donor and acceptor PBA since the energy of LUMO ${ }_{\text {nthracene }}(-0.07270$ hartree is $-1.978 \mathrm{eV})$ is greater than the energy of LUMO Pentabromoacetylene $(-0,11086$ hartree or $-3.017 \mathrm{eV}$ ).

The recombination of excitons is not possible since the energy of HOMO Anthracene (-0, 20278 hartree or $-5,518 \mathrm{eV})$ is higher than that of HOMOPentabromo acetylene $(-0.22090$ hartree or $-6,011 \mathrm{eV})$ (Figure 5). The material (s) to be introduced into a solar cell must be doped. Bromine being the best of the electronic acceptors tested in the present work and potassium the best donor, a $\mathrm{Br}$ or $\mathrm{K}$ atom was introduced into a polyacetylene molecule in order to evaluate the improvement in PBA conductivity by doping. The choice is made for tri (bromoacetylene). Figure 6 illustrates the doping of the tri (bromoacetylene) by bromine and potassium respectively. Tri (bromoacetylene) is certainly the simplest molecule on which this quantochemical test is possible; an extrapolation can then be made on 
the most complicated molecules. The lengths of the $\mathrm{CC}$ bonds of tri (bromoacetylene) and of its doped forms are given in Table 2.

On reading this table it can be seen that, in the case of bromine doping, the single and double bonds of the carbon chain have passed to intermediate lengths. This suggests that doping has enhanced electronic delocalisation in the molecule; which would lead to an elevation of the conduction of the organic material. The same conclusion is practically reached when considering potassium doping. The carbon atoms of tri (bromoacetylene) and its doped forms were analyzed. Their values are shown in Table 3 .

Table 2 Lenghts of the CC bonds of tri (bromoacetylene) and of its doped forms

\begin{tabular}{llll}
\hline & Tri (bromoacetylene) & Tri(bromoacetylene) doped by Br & Tri(bromoacetylene) doped by K \\
\hline CIC3 & $1.338 \AA$ (double) & $1.371 \AA$ & $1.357 \AA$ \\
C3C5 & $1.46 \AA$ (single) & $1.400 \AA$ & $1.41 \AA$ \\
C5C6 & $1.354 \AA$ (double) & $1.478 \AA$ & $1.395 \AA$ \\
C6C8 & $1.452 \AA$ (single) & $1.505 \AA$ & $1.398 \AA$ \\
C8C9 & $1.346 \AA$ (double) & $1.335 \AA$ & $1.378 \AA$ \\
\hline
\end{tabular}

Table 3 Mulliken charges of tri (bromoacetylene) carbon atoms and its doped forms

\begin{tabular}{llll}
\hline & Tri (bromoacetylene) & Tri (bromoacetylene) dopé Par le Br & Tri (Bromoacetylene) dopé par le K \\
\hline$C^{1}$ & $-0,135$ ua & $-0,185 \mathrm{ua}$ & $-0,31$ lua \\
$C^{3}$ & $-0,212$ ua & $-0,140 \mathrm{ua}$ & $-0,055 \mathrm{ua}$ \\
$C^{5}$ & 0,767 ua & 0,253 ua & $0,204 \mathrm{ua}$ \\
$C^{6}$ & $-0,989 \mathrm{ua}$ & $-0,405 \mathrm{ua}$ & $-0,715 \mathrm{ua}$ \\
$C^{8}$ & 0,253 ua & $0,326 \mathrm{ua}$ & $0,0516 \mathrm{ua}$ \\
$C^{9}$ & $-0,312$ ua & $-0,569 \mathrm{ua}$ & $-0,538 \mathrm{ua}$ \\
\hline
\end{tabular}

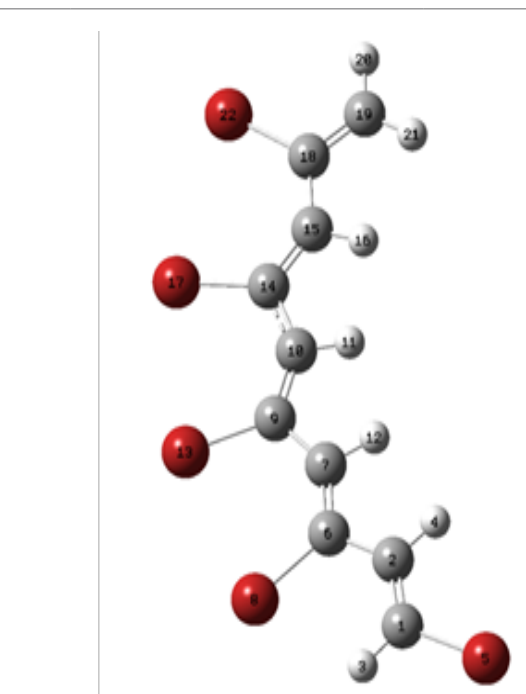

Figure 3 Molecule de pentabromoacetylene.

From the analysis of Table 3, it appears that the bromine atom has captured one of the $\pi$ C5C6 electrons; in fact, C6 was visibly depleted in electron (its charge varied from -0.989ua to -0.405ua) while C5 was enriched in electron (its charge having risen from 0.767 to $0.253 \mathrm{ua}$ ). The hole which appeared at C6 delocalized on both sides of $\mathrm{C} 6$ in accordance with the experimental observations of the literature. ${ }^{1,2}$ The same pattern occurs in the case of potassium doping with the difference that in this case the doping agent has rather yielded an electron to the organic material, thus causing an electronic surplus instead of a defect at the level of this material. These doping operations can be illustrated as in Figure 7. The doping thus causes a local jamming of the alternation between double and single bonds ${ }^{1,2}$ in the polymers of conjugated organic compounds.

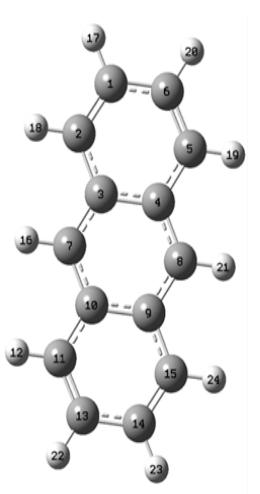

Figure 4 Molecule d'anthracène.

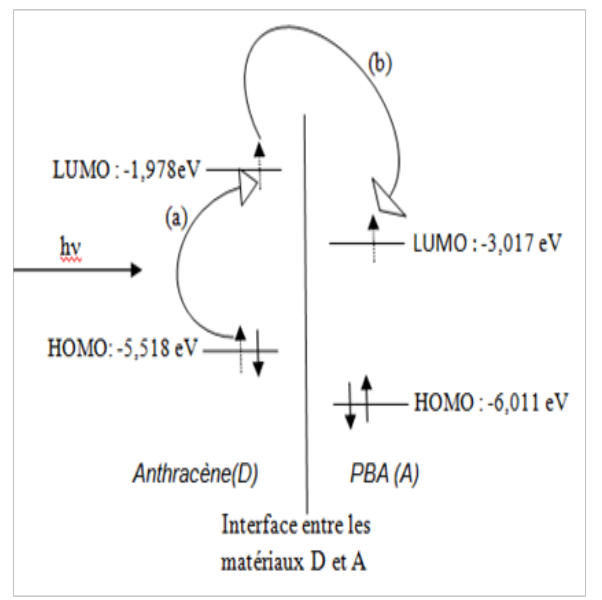

Figure 5 Mechanism of creation and dissociation of an excitons: the electron represented in dotted line passes from the HOMO of D to its LUMO (arrow a), then jumps on the LUMO of $A$ (arrow $b$ ). 
Table 4 presents energy parameters characteristic of the photoelectronic conduction of organic molecules. Table 5 provides information on the UV-Visible absorption domains of the two materials proposed, in the present work, to constitute the active phase of a photovoltaic cell; these are anthracene and pentabromoacetylene. It is interesting to note that $\mathrm{Br}$ and $\mathrm{K}$ are good doping agents. With bromine, $\mathrm{P}$ doping is carried out while N-type doping is observed with potassium. Doping with potassium is more than six times better with doping by bromine; The doping thus causes a local jamming of the alternation between double and single bonds ${ }^{1,2}$ in the polymers of conjugated organic compounds.

Table 4 Photoelectronic conduction characteristics of test molecules
In fact potassium causes the passage of the tribromoacetylene gap from 4.02 to $0.37 \mathrm{eV}$ while bromine passes this gap from 4.02 to $2.38 \mathrm{eV}$. From this observation it can be deduced that bromine and potassium can be used to pentabromoacetylene; this material can be proposed to make an anthracene/pentabromoacetylene photovoltaic cell. If necessary, the bromine will serve to the pentabromoacetylene in order to increase the holes in this material and, consequently, to reinforce its "electron acceptor" capacity; at the same time anthracene can be doped with potassium, which will increase the number of free moving electrons and, consequently, will enhance the "electron donor" capacity of this aromatic compound.

\begin{tabular}{lllll}
\hline Polyethylenes & & & & \\
\hline Molecule & LUMO (u.a.) & HOMO (u.a.) & Gap (u.a.) & Gap (eV) \\
\hline Hexatriene & -0.060 & -0.220 & 0,160 & 4,36 \\
Tribromoacetylene & -0.087 & -0.235 & 0,148 & 4,028 \\
Tribromoacetylene doped by Br & -0.128 & -0.216 & 0,088 & 2,38 \\
Tribromoacetylene doped by K & -0.110 & -0.124 & 0,013 & 0,37 \\
\hline
\end{tabular}

Table 5 UV-Visible absorption domains of anthracene and pentabromoacetylene

\begin{tabular}{llll}
\hline Molecule & Electronic transition & Wave length $(\mathbf{n m})$ & Energy $(\mathrm{eV})$ \\
\hline \multirow{3}{*}{ Anthracene } & $47 \rightarrow 48$ & 385.96 & 3.2124 \\
& $47 \rightarrow 49$ & $322.4 \mid$ & 3.8455 \\
& $47 \rightarrow 50$ & 276.07 & 4.4910 \\
Pentabromoacetylene & $|2| \rightarrow \mid 22$ & 402.28 & 3.0820 \\
& $|20 \rightarrow| 22$ & 383.49 & 3.2330 \\
& $|19 \rightarrow| 22$ & 345.96 & 3.5838
\end{tabular}
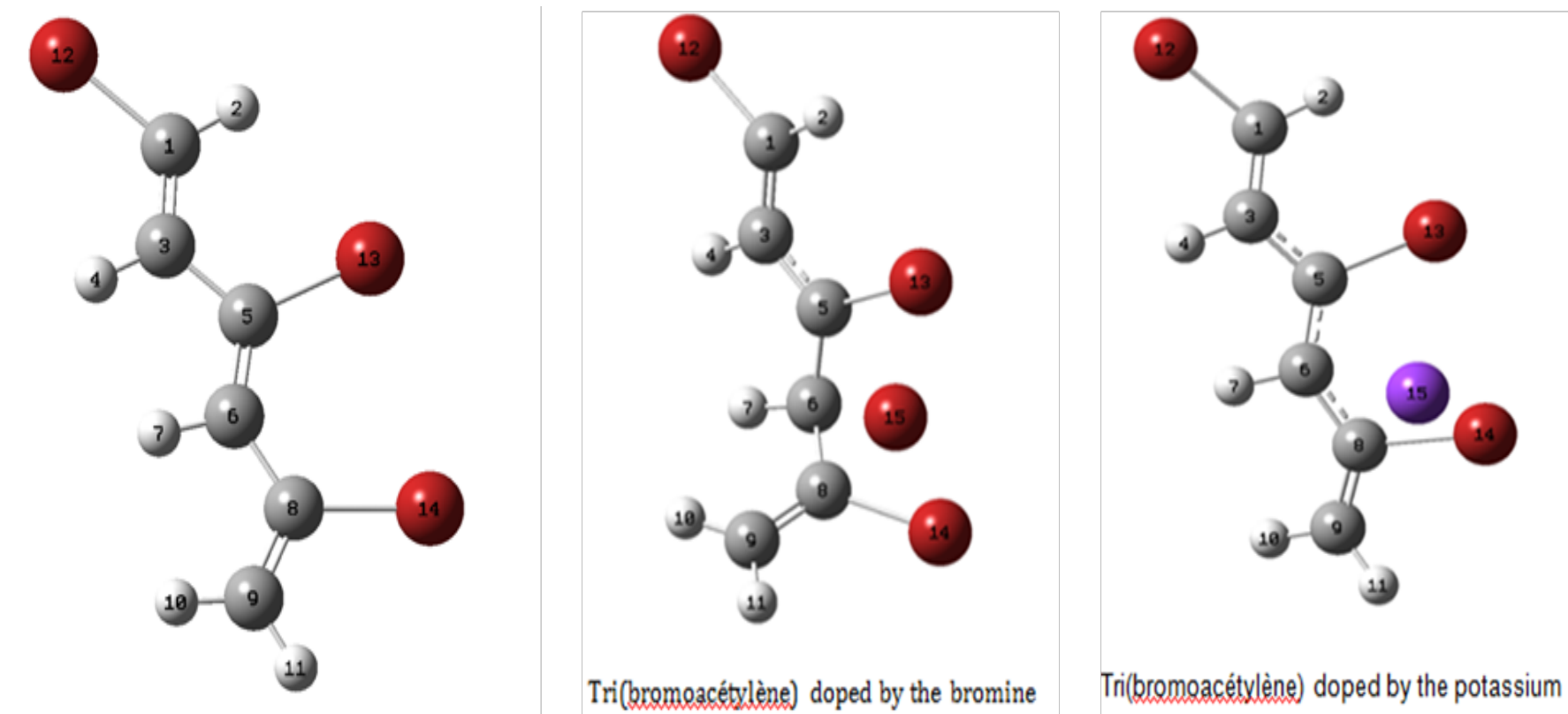

Figure 6 Doping of the tri (bromoacetylene) respectively by bromine and potassium. 


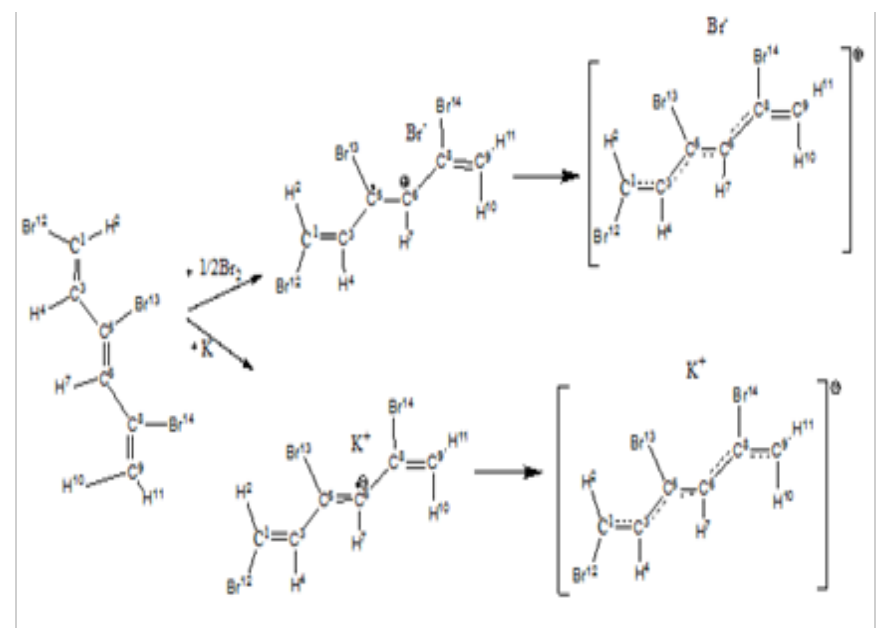

Figure 7 Doping reactions of the tri (bromoacetylene).

\section{Conclusion}

By the DFT method a study of photosensitive organic materials was carried out. As a result,

i. The electrical conduction of organic materials is promoted by the presence of conjugated double bonds in the organic molecule; this conduction is all the more marked as the number of conjugated links increases, which is reflected by the decrease in the HOMO-LUMO gap when increasing this number in a given series.

ii. The fact that these molecules absorb in the UV-visible range of the solar electromagnetic spectrum, as evidenced by the HOMO-LUMO gap values, justifies their use in photovoltaic cells.

iii. Potassium and bromine can be used to photosensitive organic materials: potassium for type $\mathrm{N}$ doping, bromine for P-type doping.

iv. A mixture of penta (bromoacetylene) doped with bromine and anthracene can serve as a photosensitive layer in a photovoltaic cell.

\section{Acknowledgements}

None.

\section{Conflicts of interest}

The author declares no conflict of interest.

\section{References}

1. Richard F. Conception, synthèse et caractérisation de polymères à blocs bâtonnet-pelote en vue d'applications photovoltä̈ques: de la macromolécule au dispositif. Strasbourg, France: ABES; 2008. p. 34 52 .

2. Leliège A, Grolleau J, Allain $M$, et al. Small $\mathrm{D}-\pi-\mathrm{A}$ systems with o-phenylene-bridged accepting units as active materials for organic photovoltaics. A European Journal. 2013;19(30):9948-9960.

3. Yinhua Z, Canek F, Talha M Kh, et al. Recyclable organic solar cells on cellulose nanocrystal substrates. Sci Rep. 2013;3:1536.

4. De Cuendias A. Copolymères à blocs constitués d'un bloc rigide conjugué: synthèse et auto-assemblage en solution. Thèse de doctorat, Bordeaux, France: Université ; 2017. p. 6-58.

5. Monestier F. Amélioration de l'absorption photonique dans les cellules photovoltä̈ques organiques. Thèse de doctorat, France: Université Paul Cezanne Aix-Marseille III ; 2008. p. 35-40.

6. Kamino BA, Mills B, Reali C, et al. Liquid triarylamines: the scope and limitations of piers-rubinsztajn conditions for obtaining triarylaminesiloxane hybrid materials. J Org Chem. 2012;77(4):1663-1674.

7. Merad Boudia MR. Confinement Effect on the Dewetting of Polymer Thin Films. Doctoral thesis, University Abou Bekr Belkaid (Tlemcen, Algeria); 2012.

8. Mathew Clark. Introduction to theoretical organic chemistry and molecular modeling. William B Smith. J Chem Inf Comput Sci. 1997;37(3):625.

9. Chaquin P. Manuel de chimie théorique. Application à la structure et à la réactivité en chimie moléculaire. France: Ellipses; 2000. 189 p.

10. Becke AD. Density functional thermochemistry. III. The role of exact exchange. J Chem Phys. 1993;98:5648.

11. Lee C, Yang W, Parr RG. Development of the Colle-Salvetti correlation-energy formula into a functional of the electron density. Phys Rev B. $1980 ; 37: 785$.

12. Nadjia L. Thèse de doctorat es-sciences. Algérie: Université de Bartna; 2009.

13. Frisch MJ. Gaussian 98W. Pittsbourgh PA, USA: Gaussian Inc; 1998.

14. Hehre WJ, Radom L, S Chleyer PVR, et al. Ab initio molecular orbital theory. JCC. 1986;7(3):379.

15. C haquin T. Pratique de la chimie Théorique, Inter Editions ; 1995.

16. Melius CF, Goddard WA. Phys Rev A. 1974;10:1528-1540.

17. Kahn LR, Baybutt P, Truhlar DG. J Chem Phys. 1976;65:3826-3853.

18. Becke AD. Density-functional thermo chemistry. III. The role of exact exchange. J Chem Phys. 1993;98(7):5648-5652. 